\title{
Promoting HIV Testing Through Non-Governmental Organizations in China: Complementary to the Government Programs
}

\author{
Huey T Chen ${ }^{1,2 *}$, Wei Fang ${ }^{2}$, Nanette C Turner ${ }^{1}$ and Han-Zhu Qian ${ }^{3,4}$ \\ ${ }^{1}$ Department of Public Health, Mercer University, Atlanta, Georgia, USA \\ ${ }^{2}$ Zhejiang University of Technology, Zhejiang, China \\ ${ }^{3}$ Vanderbilt Institute for Global Health, USA \\ ${ }^{4}$ Division of Epidemiology, Vanderbilt University, Nashville, Tennessee, USA
}

\section{Government-Based HIV Testing in China}

Human immunodeficiency virus (HIV) voluntary counseling and test (VCT) is an intervention that includes both voluntary pre- and post-test counseling and HIV testing. Its essence is that people, of their own free will, opt for VCT, and it provides them with an opportunity to confidentially explore and understand their HIV risks as well as to learn their HIV status. The purpose of VCT is to improve access to prevention, care and treatment services through early detection of HIV [1]. Early detection, timely linkage to care, and prompt initiation of antiretroviral therapy (ART) also have additional benefits in reducing HIV transmission as well as morbidity and mortality $[2,3]$. VCT in many countries is provided by non-governmental organizations (NGOs).

In China, VCT services are mainly delivered through government programs. There are two government sponsored service networks in China: VCT clinics in China's Center for Disease Control and Prevention (CDCs) and public hospitals [4]. Over 9000 VCT sites have been established in CDCs which offer free VCT at the province, city and county level. Alternatively, people can receive HIV testing in public hospitals with fees.

The Chinese government-based VCT programs allow the government to effectively monitor the implementation of VCT and assure that VCT practices are aligning well with the national policies for combating the HIV/AIDS epidemic. However, the governmentbased VCT also has drawbacks. Individuals at high risks may lack trust in these programs and be reluctant to use the services due to concerns about personal security or discrimination $[5,6]$. High risk individuals may also find that the clinic times are inconvenient. As a consequence, the government-based VCT sites are not adequately reaching the majority of high risk groups. For example, among men who have sex with men (MSM), the most at risk group in China, the average rate of lifetime HIV testing during 2000 and 2006 was $24 \%$, and was $47 \%$ during 2007 and 2011; the 12 month testing rates were even lower, $21 \%$ and $38 \%$ respectfully [7].

When a large proportion of high risk populations do not know their HIV status, China misses opportunities for effectively combating the HIV/AIDS epidemic from several fronts. The knowledge of HIV infection status relates to the reduction of risky sexual behaviors among persons who test seropositive (8). When people with AIDS know their HIV status, they are more likely to take precautions during high risk interactions that may result in infecting others. In addition, VCT provides a counseling linkage on risk reduction, referral to care, and initiation of ART when indicated [1-3]. Early diagnosis and treatment is a crucial element in reducing the transmission to seronegative partners $[8,9]$.

Furthermore, government sites in China tend not to perform VCT according to its principles and spirit. A study by $\mathrm{Wu}$ and his associates provided an interesting discussion on HIV testing policy and implementation in China [10]. According to the study, the governmental policy was to actively identify infected individuals by sending government health workers to recruit high risk populations for HIV testing in communities and institutional settings, such as prisons, and put the HIV positive persons in contact with treatment services. The intention of the policy was noble, but the implementation presented several drawbacks. This study used Yunnan province's experience as an example to illustrate the point. The government of Yunnan province launched a HIV testing initiative from September to December 2004. They invited 424,000 high risk individuals to be tested, of whom $1.3 \%$ declined. Of those participating in the testing, $3.2 \%$ were positive for HIV. The policy, theoretically, followed the model of VCT in principle, but not in its actual implementation. HIV testing was often carried out without the individual's voluntary pre-test and post-test counseling. Since the active testing may involve little counseling and may not always be voluntary, the concern of whether the active testing of high risk groups violated human rights was also raised [11]. Finding alternative and creative strategies or policies for providing better VCT services to high risk populations is an urgent issue for HIV prevention in China [4].

\section{Potential Benefits of NGO-Based VCT at China}

A natural way to search for alternative strategies for delivering VCT is to look at VCT programs and experiences implemented in other countries. In many developed countries (including the United States and European countries) and developing countries (including India, Thailand, Brazil, and many African nations), non-government organizations (NGOs) are major VCT providers and serve as a bridging role of linking government resources to at-risk communities [12,13]. With NGOs' partnerships, the government can place a greater emphasis and effort on performing functions such as HIV/AIDS surveillance, providing affordable AIDS care for those financially disadvantaged, initiating broad HIV/AIDS education campaigns for the public, and developing national strategic plans for HIV prevention.

Since many countries use NGOs for delivering VCT, a reasonable strategy seems to suggest that China should follow a similar path. The NGO-based VCT program has several potential advantages. Such a program would effectively reach high risk populations including

*Corresponding author: Huey T Chen, Professor, Department of Public Health Director, Mercer University Atlanta Campus, USA, Tel: 6785476725; E-mail: chen_h@mercer.edu

Received January 20, 2015; Accepted February 20, 2015; Published February 28,2015

Citation: Chen HT, Fang W, Qian HZ, Turner NC (2015) Promoting HIV Testing Through Non-Governmental Organizations in China: Complementary to the Government Programs. J AIDS Clin Res 6: e117. doi:10.4172/2155-6113.1000e117

Copyright: $\odot 2015$ Chen HT, et al. This is an open-access article distributed under the terms of the Creative Commons Attribution License, which permits unrestricted use, distribution, and reproduction in any medium, provided the original author and source are credited. 
injection drug users, sex workers, migrants, street children, and other disadvantaged segments of the community. These high risk populations are often highly marginalized and stigmatized people, resulting in fearful or distrustful views of government officials, including government health workers; thus making it difficult for government workers to reach high risk populations. On the other hand, NGO staff would be regarded by high risk populations as non-threatening. As a result, high risk populations would have less concern for being stigmatized and discriminated if the VCT programs are organized by NGOs.

Furthermore, it is quite possible that government health workers in China are already overwhelmed by their routine disease prevention and control works. This may be one of the major reasons why they do not currently provide time-consuming counseling services to high risk populations when they perform HIV testing. The use of NGOs as a new portal for providing VCT may be able to resolve the controversies related to the current HIV testing policy, such as government workers possibly using coercion to recruit high risk populations for testing or that HIV testing is administered without counseling.

Currently there are two types of NGOs in China: Grass-root and government-supported. These two types of NGOs are rather different in terms of financial sources, autonomy, and relationships with the government and community. Any discussion of NGOs in China would be perplexing or even misleading without a distinction of these two. The rest of the article will highlight differences between these two types of NGOs and discuss the prospect of utilizing each one of them to deliver VCT under the political, legal, and institutional context of Chinese society.

\section{Prospect for Using Grass-Root NGOs for delivering VCT}

Chinese grass-root NGOs are similar to community-based organizations (CBOs) in Western societies with the following key features: autonomous, private, non-profit-distributing, self-governing, and voluntary $[12,13]$. These NGOs are established and managed by the community members, and therefore, have good trusts and connections with the targeted populations, and could potentially better serve the community. However, many of these NGOs in China are not formally recognized by the government. The Chinese government tends to view these kinds of autonomous organizations as a potential threat to internal security and national interest; mainly because they do not follow the government's protocol. With such a concern, the government applies many institutional and legal barriers for establishing a NGO. It is very difficult for a social organization to obtain a legal NGO status. In spite of the hurdles, grass-root NGOs have emerged largely over the past decades. A recent study [14] used web search and snow-ball procedures was able to identify 263 grass-root NGOs in three provinces/ municipality: Yunnan, Beijing, and Guangdong. These organizations were completely unregistered, registered for profit business, registered in Hong Kong, or claimed to be sponsored under another organization. They provide services in areas such as HIV/AIDS, labor rights, the environment or education. Many of these organizations have received supporting funds from International NGOs and foundations. For example, Red Cross Australia, UNAIDS, and Save the Children have HIV/AIDS related projects in Yunan. Similarly, private foundations such as Ford Foundation and Asia Foundation have funded HIV prevention programs since 1990s [15]. These international NGOs and private foundations have managed to work with local NGOs to provide services to high risk groups. Grass-root NGOs have made contributions to disseminating HIV/AIDS prevention and treatment knowledge in addition to providing health training. An example of this kind of NGO is Shanghai Leyi [16]. This NGO provides HIV/AIDS education and legal services to female and male sex workers in Shanghai. Shanghai CDC has difficulty reaching these high risk populations. Shanghai Leyi is registered as a private company, emulating organizational structure and decision making of western NGOs. It has also collaborated with UNAIDS on HIV/AID project.

However, the total financial and human resources of these grassroot NGOs are slim relative to Chinese populations and communities [14-16]. With a combination of the resource restriction with all the other political, legal, and institutional barriers discussed above, the feasibility and capability of using these autonomous grass-root NGOs as one of the major partners for providing VCT services for Chinese populations and communities is severely limited.

\section{Prospect of Using Government-Supported NGOs to Deliver VCT}

The government-sponsored NGOs have existed since the establishment of new China in 1949, but they may still be unfamiliar to many westerners. Some examples of these NGOs are the Chinese Labor Union, the Chinese Family Planning Association, Chinese Women's Federation, Chinese Red Cross Society, and Chinese Youth League. The political, social and cultural backgrounds for having this kind of NGOs are as follows: Chinese tend to view the autonomy of NGOs not as a yes or no distinction, but as a matter of degree. If autonomy is a degree, then China has a vast number of Chinese-style NGOs at local and national levels. This kind of NGO is frequently called "governmentsupported" or "Chinese" NGO for distinguishing their differences from western style NGOs [17]. The government-supported NGOs are notfor-profit organizations, financially supported by the government. Some government officials may serve as the leaders of these NGOs after they retired from government positions, resulting in the continuance of strong relationships with the government and opportunities for further financial support. These NGOs contribute to the public welfare and are of service to society. They are well-established organizations serving as bridges to link the government and society in community affairs. These NGOs have their own local organizations and staff in each community, are well connected with other local governmental organizations, work closely with community residents, and have been regarded as part of the social fabric of Chinese society since the Communist Revolution.

With the economic reform and social changes from the last several decades, these NGOs are seeking new missions for renewal of their organizations and now having the Chinese government's support and encouragement to engage in HIV prevention activities [18]. For example, The Global Fund to Fight AIDS, Tuberculosis and Malaria (GFATM) sponsors the China CARES (Comprehensive AIDS Response) program. The funds go directly to the Chinese Center of Disease Control and Prevention. GFATM asked the Center to find and fund local NGOs to provide HIV prevention services such as outreach and education to high risk populations such as migrants and impoverished people. It is of little surprise that the Center has allocated the great majority of the funds to government-support NGOs rather than grass-root NGOs.

The experience of China CARES program demonstrates that not only does the Chinese government advocate for governmentsupported NGOs to do HIV prevention, but it also indicates that these organizations are interested in and capable of delivering HIV prevention in areas of HIV education and training. With many hindrances for using grass-root NGOs for delivering VCT as discussed in the last section, 
these government-supported NGOs may be an excellent candidate for taking on the VCT mission in China, because governmentsupported NGOs work in the community and are familiar with the local population. Government-supported NGOs communicate well with residents and have a better chance to reach high risk populations when compared to other government agencies. Furthermore, not only are these organizations legal in China, but they are also trusted and have excellent connections with the Chinese government. In addition to the above benefits, government-supported NGOs have offices and staff in every community. With their manpower and connection with the government, they would be an excellent candidate for establishing alternative VCT sites that compensates the limitations of CDC VCT sites for serving high risk populations across communities in China. Overall, these government-supported NGOs may be more suitable than autonomous grass-root NGOs in delivering VCT.

However, the above discussions are based in theory. To be persuasive, empirical evidence on the following questions are still needed: Could these government-supported NGOs effectively reach high risk populations? Could they provide high-quality VCT to them? A pilot study [19] of a government-supported NGO-based VCT program provides direct evidence for answering these questions. This pilot study focused on serving injection drug users (IDUs) in the southern city of Guangxi. The city is adjacent to Vietnam and on a major drug traffic route from the Golden Triangle to major cities in China. The government-supported NGO in the project was a local Women's Federation. This project closely followed VCT's "voluntary" principle rather than the typical government VCT practice as discussed above. In this project, NGO staff was trained to deliver VCT by using a VCT protocol targeting injection drug users developed in the United States. The model had standard pre-test counseling and post-test counseling components. Due to their satisfaction with services, many participants volunteered to bring other IDUs to join the program. The NGO recruited 226 male and female IDUs in 4 months. Approximately $85 \%$ of the participants were male. The data from the participants' exit survey and focus group meetings clearly indicated that they were reluctant to go to government VCT sites, but were receptive to the VCT site run by the government-supported NGO. These participants engaged in high risk behaviors, with $63.3 \%$ reported to have some type of injection use risk behavior including sharing needles or using weak cleaning solutions. Thirty-nine percent reported reuse of other's needles in the previous 30 days and $82 \%$ of those sexually active reported never using condoms during intercourse with non-main partners. The data also indicated that none used proper sterile cleaning techniques for needles and syringes. The HIV prevalence rate of the group was as high as $57.5 \%$. A comparison between the baseline and three month follow-up data showed that the intervention substantially increased IDUs' HIV/AIDS knowledge, reduced sharing needles/syringes, and increased condom use. This study provides convincing evidence that with proper training, a government-supported NGO is capable of effectively reaching high risk populations and delivering a standard VCT to high risk population with desirable outcomes.

Another lesson learned from this project is that a governmentsupported NGO VCT program has little difficulty for winning support from local government agencies including the local CDC because of their existing working relationships. In this project, the Women's Federation developed strong collaborations with city government, local $\mathrm{CDC}$, and hospitals for assuring that clients were properly referred to services and that the confidentiality and safety of clients were protected. Government-supported NGOs have excellent social capitals with government organizations in a community. These capitals would be an asset for building a supportive network for a successful VCT program.

\section{Conclusion and Discussion}

This study provides justifications and some evidence to suggest that government-supported NGOs have a potential for scaling up VCT for serving high HIV risk population in China. In order to facilitate this happen, an enabling environment must be created first. The study proposes the following strategies to promote government-support NGOs as one of the key partners for providing VCT services:

\section{Make clear which type of NGOs the government refers to when they make official policy announcements and statements}

An interesting development is that the Chinese government has made increasing public acknowledgements for the need to include NGOs in HIV prevention. For example, the Ministry of Health of China has repeatedly made policy statements that a "full use of NGO work" is one of the key elements for fighting HIV/AIDS in China $[20,21]$. These acknowledgements are very encouraging. However, based upon the above discussions, it would be much more helpful if the government could clarify the following question: Which types of NGOs are to be fully used? Would they be grass-root NGOs, governmentsupported NGOs or both? Or, which types of NGOs go first? If the government could clarify this issue, it would provide a guide for public health agencies, security agencies, academia, domestic partners, and international partners to use. This may facilitate them to work together for systematically developing more sustainable VCT and other HIV/ AIDS prevention programs for more effectively combating HIV/AIDS epidemic in the future.

Facilitate dialogues and collaborations between the public security sector and public health sector on issues such as how to deal with high risk populations

In spite of progress, there is a tension between the public security sector and public health sector regarding how to deal with high risk populations like drug users and sex workers. The public security sector views drug users and sex workers primarily as criminals who should be jailed, punished, and re-educated. On the other hand, health professionals believe services need to be provided to drug users and sex workers to reduce their HIV risk behaviors. The tension could slow the path for expanding HIV prevention efforts and developing innovative programs such as the NGO-based VCT program. In spite of the barriers, there are signs of encouragement. The public security sector is working with the public health sector in implementing harm reduction strategies, such as methadone programs and needle exchange programs for IDUs in some areas. The acceptance of harm reduction philosophies in both sectors should pave a way for justifying government-support NGO roles in HIV prevention, including delivering VCT.

\section{Encouraging studies in government-supported NGO VCT programs}

Up-to-date, our literature search found only one empirical study on the government-support NGO VCT program. In order to effectively promote this HIV testing mode, more studies are needed to demonstrate the feasibility and merits of using the government-support NGO VCT program as a complementary, viable force for the existing government HIV testing program.

\section{Create HIV prevention funding specific for supporting government-support NGO based VCT programs}

Currently, the government only funds CDC-based VCT sites in an ongoing basis. Finding a way to persuade the government to fund 
Citation: Chen HT, Fang W, Qian HZ, Turner NC (2015) Promoting HIV Testing Through Non-Governmental Organizations in China: Complementary to the Government Programs. J AIDS Clin Res 6: e117. doi:10.4172/2155-6113.1000e117

Page 4 of 4

government support NGO-based VCT will be a challenge. More research demonstrating effectiveness and viability of governmentsupport NGO-based VCT programs may create a momentum for attracting government and/or international funding organizations attention and support for such a program.

\section{References}

1. World Health Organisation/Joint United Nations Programme on HIV and AIDS (2007) Guidance on Provider-Initiated HIV Testing and Counselling in Health Facilities, Geneva: World Health Organisation.

2. Gardner EM, McLees MP, Steiner JF, Del Rio C, Burman WJ (2011) The spectrum of engagement in HIV care and its relevance to test-and-treat strategies for prevention of HIV infection. Clin Infect Dis 52: 793-800.

3. Cohen MS, Chen YQ, McCauley M, Gamble T, Hosseinipour MC, et al. (2011) Prevention of HIV-1 infection with early antiretroviral therapy. N Engl J Med 365: 493-505.

4. Tao J, Li MY, Qian HZ, Wang LJ, Zhang Z, et al. (2014) Home-based HIV testing for men who have sex with men in China: a novel community-based partnership to complement government programs. Plos One 9: e102812.

5. Han-Zhu Qian, Joseph E Schumacher, Huey T Chen, Yu-Hua Ruan (2006) Injection drug use and HIVIAIDS in China: review of current situation, prevention and policy implications. Harm Reduct J 3:4.

6. State Council AIDS Working Committee and UN Theme Group on HIVIAIDS in China (2004) A joint assessment of HIVIAIDS prevention, treatment, and care in China, 2004, China Ministery of Health, Beijing.

7. Zou H, Hu N, Xin Q, Beck J (2012) HIV testing among men who have sex with men in China: a systematic review and meta-analysis. AIDS Behav 16: $1717-1728$.

8. Zakher B, Blazina I, Chou R (2014) Association between knowledge of HIVpositive status or use of antiretroviral therapy and high-risk transmission behaviors: systematic review. AIDS Care 26: 514-521.
9. Vermund SH, Hayes RJ (2013) Combination prevention: new hope for stopping the epidemic. Curr HIVIAIDS Rep 10: 169-186.

10. Wu Z, Sun X, Sullivan SG, Detels R (2006) Public health. HIV testing in China Science 312: 1475-1476.

11. UNAIDS-WHO (2005) Policy statement on HIV testing.

12. Crane SF, Carswell JW (1992) A review and assessment of non-governmental organization-based STD/AIDS education and prevention projects for marginalized groups. Health Educ Res 7: 175-194.

13. Mercer MA, Liskin L, Scott SJ (1991) The role of non-governmental organizations in the global response to AIDS. AIDS Care 3: 265-270.

14. Spires AJ, Tao L, Chen KM (2014) Societal social Support for China's grass Roots NGOs: Evidence from Yunan, Gangdong, and Beijing. China Journa 71: $65-80$.

15. Bonner S, Burgess S, Dent S, Lehnen C, Yates M (2011) Combating HIV-AIDS in China: Is there a place for NGOs.

16. Quusha Ma (2002) Defining Chinese Nongovernmental Organizations. Voluntas 13: $113-130$

17. Quusha Ma (2006) Non-Governmental Organizations in Contemporary China Paving the way to civic society? Routledge, New York.

18. Xu H, Zeng Y, Anderson AF (2005) Chinese NGOs in action against HIVIAIDS Cell Res 15: 914-918.

19. Chen HT, Liang S, Liao Q, Wang S, Schumacher JE, et al. (2007) HIV voluntary counseling and testing among injection drug users in south China: a study of a non-government organization based program. AIDS Behav 11: 778-788.

20. Ministry of Health of China, UNAIDS, WHO (2006) 2005 Update on the HIVI AODS epidemic and response in China. Ministry of Health of China: Beijing.

21. Xinhua (2012) China's NGOs praised for role in fighting HIV/AIDs. 